\title{
Exploiting Material Resonances to Reduce Losses in Plasmonic Modulators
}

\section{Conference Paper}

\section{Author(s):}

Haffner, Christian; Heni, Wolfgang; Chelladurai, Daniel (D); Dordevic, Nikola (D); Koch, Ueli (D); Fedoryshyn, Yuriy M.; Portner, Kevin; Burla, Maurizio; Elder, Delwin L.; Robinson, Bruce H.; Dalton, Larry R.; Leuthold, Juerg (D)

Publication date:

2017

Permanent link:

https://doi.org/10.3929/ethz-b-000221556

Rights / license:

In Copyright - Non-Commercial Use Permitted

Originally published in:

https://doi.org/10.1364/NOMA.2017.NoM2C.3 


\title{
Exploiting Material Resonances to Reduce Losses in Plasmonic Modulators
}

\author{
C. Haffner ${ }^{1 *}$, W. Heni ${ }^{1}$, D. Chelladurai ${ }^{1}$, N. Dordevic ${ }^{1}$, U. Koch ${ }^{1}$, Y. Fedoryshyn ${ }^{1}$, K. Portner ${ }^{1}$, M. Burla ${ }^{1}$, \\ D. L. Elder ${ }^{1}$, B. Robinson ${ }^{2}$, L. R. Dalton ${ }^{2}$ and J. Leuthold ${ }^{1}$ \\ ${ }^{1}$ Institute of Electromagnetic Fields (IEF), ETH Zurich, Zurich, Switzerland \\ ${ }^{2}$ Department of Chemistry, University of Washington, Seattle, WA 98195-1700, United States \\ *haffnerc@ethz.ch
}

\begin{abstract}
We demonstrate a two times reduction of plasmonic modulator loss by exploiting material resonances of organic electro-optic materials. We measure enhanced in-device electro-optic coefficients with record values of $r_{33}=325 \mathrm{pm} / \mathrm{V}$ reducing $U_{\pi} L$ threefold.

OCIS codes: (250.5403) Plasmonics; (230.2090) Electro-optical devices; (190.4710) Optical Nonlinearities in organic material; (250.4110) Modulators;
\end{abstract}

\section{Introduction}

Electro-optic (EO) materials, such as inorganic [1] or poled organic [2] ones, are the materials of choice for EO modulators as the Pockels effect provides one of the most efficient and fastest gateways for linearly encoding an electrical signal onto the phase of an optical carrier. The performance of these modulators is determined by the strength of the material's EO coefficient $r$ and large values of this parameter are required to realize compact, energy efficient and fast modulators that can be effectively co-integrated with electronics [3].

Most of the commercially employed modulators are based on lithium niobate $\left(\mathrm{LiNbO}_{3}\right)\left(r_{33}=34 \mathrm{pm} / \mathrm{V}\right.$ [1]). However, the large difference of the optical and RF permittivity results in a walk-off between the co-propagating electrical and optical signals, ultimately limiting the operation speed. In contrast, organic electro-optic (OEO) materials $\left(r_{33}>250 \mathrm{pm} / \mathrm{V}\right)$ are designed to overcome this obstacle, allowing one to encode THz signals onto an optical carrier [4]. Applying either material system within weakly guided modulators results in a voltage-length product on the order of $\mathrm{Vcm}$ and $\mathrm{cm}$-long devices. Incorporating $\mathrm{LiNbO}_{3}$ and $\mathrm{OEO}$ materials into modulators based on high refractive index photonic waveguides has enabled device lengths of millimeters and even sub-millimeters [5,6]. Yet, downscaling to microns requires alternative approaches, like plasmonics which enable sub-diffraction light confinement at the expense of ohmic losses [7]. The losses are on the order of $\mathrm{dB} / \mu \mathrm{m}$ so the strength of the lightmatter interaction needs to overcome a certain threshold to be able to encode the electrical signal within a few microns [8]. This poses strict requirements onto the strength of the EO-coefficient $(r>100 \mathrm{pm} / \mathrm{V})$ to realize a phase modulator with $5 \mathrm{~dB}$ insertion losses and moderate driving voltages $\left(U_{\pi}=5 \mathrm{~V}\right)[9,10]$. However, even larger $r_{33}$ values are desired to unleash the full power of low-loss, high-speed and energy efficient plasmonic modulators.

In this paper we demonstrate for the first time that modulator losses and/or driving voltage can be reduced by a factor of 2, when operating plasmonic modulators in close vicinity to the OEO material's absorption resonance. This is achieved by harnessing the resonantly-enhanced electro-optic coefficient, resulting in record-high - to the best of our knowledge-in-device EO coefficients of up to $325 \mathrm{pm} / \mathrm{V}$. Further, the enhanced light matter interaction translates to a three times smaller voltage-length product $\left(U_{\pi} L\right)$ when operating near resonance rather than off-resonance.

\section{A path to in-device electro-optic coefficients beyond $300 \mathrm{pm} / \mathrm{V}$}

The electro-optic coefficient in OEO-materials is caused by the light interaction with the $\pi$-electrons of organic molecules [2]. Due to the strong dipole moment of the organic molecules the electrons experience a strongly asymmetric potential, resulting in large second-order nonlinearities $\left(r_{33}\right)$. Furthermore, transition of the $\pi$-electrons from their ground to their excited state are typically in the visible and near IR range [2]. This transition provides a built-in resonance that increases the nonlinear light-matter interaction between $\pi$-electrons, an externally applied electrical RF-field and photons when the photon energy approaches the transition energy. However, absorption also starts to play a role and the optimum ratio between absorption and light-matter interaction is not necessarily found near resonance, but rather off resonance [11]. In ref. [11] a lossless waveguide is assumed which does not hold for plasmonic modulators having waveguide losses on the order of $\mathrm{dB} / \mu \mathrm{m}$.

Fig. 1(a) shows a top-view of a plasmonic phase modulator (PPM). Two gold contact pads form a plasmonic metalinsulator-metal slot waveguide with typical lengths ranging from 5 to $50 \mu \mathrm{m}$. Silicon waveguides are used to access the modulator on chip, while a taper coupler converts light to the plasmonic mode and vice versa [12]. Fig. 1(b) shows the cross-section of the plasmonic slot waveguide. The slot has a height of $150 \mathrm{~nm}$, is typically $50 \mathrm{~nm}$ to $200 \mathrm{~nm}$ wide and is filled with an OEO material. 
(a)

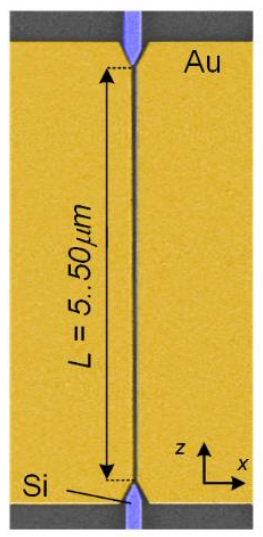

(b)

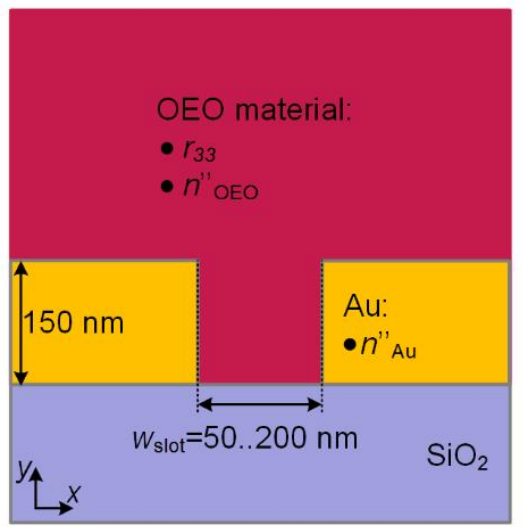

(c)

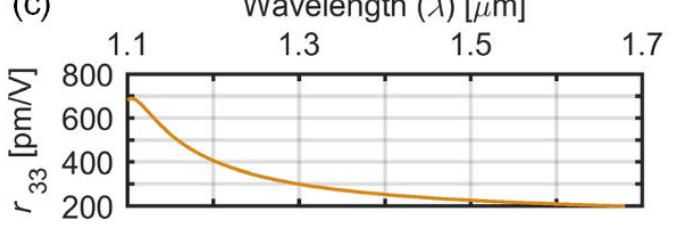

(d)

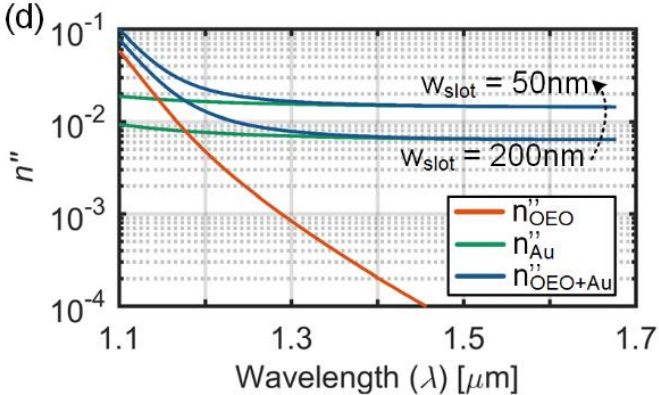

Fig. 1(a) Top view of a plasmonic phase modulator. Two gold contact pads form a plasmonic slot waveguide. (b) Cross view of the plasmonic slot waveguide. The slot is filled with an organic electro-optic (OEO) material which provides the electro-optic coefficient $\left(r_{33}\right)$ necessary for phase modulation. (c) Dispersion of $r_{33}$ over the wavelength due to material resonances. The material resonances at $1075 \mathrm{~nm}$ result in a 3.5 fold enhancement of $r_{33}$ reaching values of $700 \mathrm{pmV}$. (d) Loss $(n$ ") contribution as a function of the wavelength. The intrinsic losses of the organic material increase exponentially (red), while the plasmonic losses (green) stay almost constant. The blue lines show the total device losses. The total losses are dominated by the OEO losses for wavelengths smaller than $1.2 \mu \mathrm{m}$ and $1.25 \mu \mathrm{m}$ for slot widths of $50 \mathrm{~nm}$ and $200 \mathrm{~nm}$, respectively. These points are also defining the optimal operation point of plasmonic modulators resulting in a two times increased $r_{33}$. This allows to reduce overall losses despite the increased OEO losses.

The efficiency $\eta$ of Pockels (POH) modulators is given by the ratio of the change in the effective refractive index $\Delta n^{\prime}$ eff $(\lambda)$ and the imaginary part of the effective refractive index $\left(n{ }^{\prime \prime}\right.$ eff $\left.(\lambda)\right)$ [3]. The former component corresponds to the phase change per length, while the latter one defines the propagation loss. For a qualitative discussion we can approximate the efficiency by

$$
\eta(\lambda)=\frac{\Delta n_{\mathrm{eff}}^{\prime}(\lambda)}{n_{\mathrm{eff}}^{\prime \prime}(\lambda)} \approx \frac{n_{\mathrm{OEO}}^{\prime}(\lambda)^{3} \cdot r_{33}(\lambda)}{\left(n_{\mathrm{OEO}}^{\prime \prime}(\lambda)+n_{\mathrm{Plasmonic}}^{\prime \prime}(\lambda)\right)},
$$

where $n_{\mathrm{OEO}}^{\prime}(\lambda)$ is the real part of the refractive index of the OEO material and $n_{\mathrm{OEO}}^{\prime \prime}(\lambda) n_{\text {Plasmonic }}^{\prime \prime}(\lambda)$ are the materials' and waveguides imaginary parts. The imaginary part allows to estimate the losses of the propagating plasmonic mode. Fig. 1(c) plots the normalized theoretical electro-optic coefficient of the OEO-material (a binary chromophore composed of 75\% HD-BB-OH and 25\% YLD-124 [13]) over the wavelength calculated based on a twostate model [11]. An enhancement of $r_{33}$ by a factor of $3.5 \mathrm{can}$ be observed. This is due to the material resonance having a transition energy corresponding to $1075 \mathrm{~nm}$. The absorption of the OEO-material $\left(n\right.$ " $\left.{ }_{\text {eff }}(\lambda)\right)$ increases exponentially over the same wavelength range, see red line in Fig. 1(d). In contrast, the plasmonic losses of a $50 \mathrm{~nm}$ and $200 \mathrm{~nm}$ wide slot stay almost constant over the wavelength range of interest, see green lines Fig. 1(d). The total losses (blue line) increase exponentially only for wavelengths shorter than $1.2 \mu \mathrm{m}$, when the OEO absorption starts to dominate. The optimal operation wavelength of $\mathrm{POH}$ modulators is reached shortly before the exponential increase of loss kicks in. This results in an up to two times enhanced $r_{33}$ and allows to reduce the overall losses by the same factor as phase modulators can be made two times shorter.

\section{Influence on device performance}

The following, experiments have been performed to confirm the enhancement of the efficiency $\eta$. Light from two tunable lasers (tunable over the ranges $1260 \mathrm{~nm}-1370 \mathrm{~nm}$ and $1460 \mathrm{~nm}-1630 \mathrm{~nm}$, respectively) have been fed to a PPM with a slot width of $200 \mathrm{~nm}$, while applying a $40 \mathrm{GHz}$ RF signal. An optical spectrum analyzer (OSA) recorded the spectrum of the modulated light to determine $U_{\pi} L, r_{33}$ and loss-voltage-length product. We fabricated the PPM inhouse. The OEO material was poled slightly above its glass transition temperature $\left(110^{\circ} \mathrm{C}\right)$ with a $180 \mathrm{~V} \mu \mathrm{m}$ strong poling field. For details on fabrication and poling we refer to ref. [9].

Fig. 2(a), (b) and (c) show the measured $U_{\pi} L, r_{33}$ and loss-voltage-length $\left(\alpha U_{\pi} L\right)$ product, respectively. Pleaes note, losses are given in $\mathrm{dB}$ per length. The blue dots represents the measured values (blue dots) as a function of the wavelength, while the dashed red line highlights numerical results based on the two-state model and modal waveguide analysis [2]. 
(a)

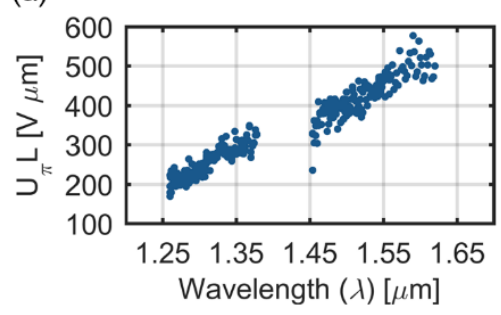

(b)

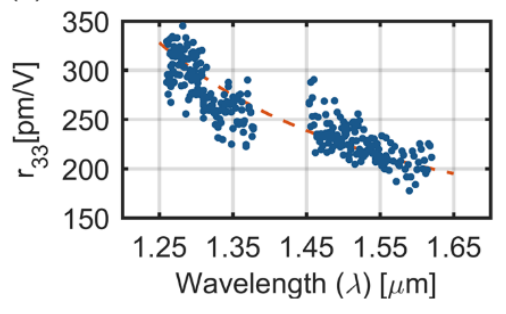

(c)

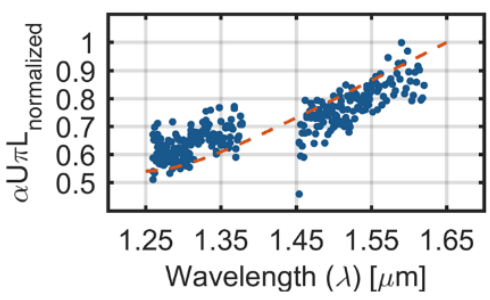

\section{- Experiment -.-Simulation/Analytic Fit}

Fig. 2 The measured (blue dots) voltage-length product (a), electro-optic coefficient (b) and loss-voltage-length product (c) as a function of wavelength. The voltage length product of the plasmonic phase modulator drops by a factor of three, mostly due to the increasing $r_{33}$ reaching indevice values of $325 \mathrm{pm} / \mathrm{V}$. The smaller voltage-length product allows a reduction of the modulator's length resulting in two times reduction of the overall losses.

The voltage-length product decreases roughly by a factor three over the wavelength range of study and is in good agreement with exact numerical studies including all effects such as plasmonic dispersion. The measurement shows an increase by $70 \%$ when reducing the wavelength and we measured - to the best of our knowledge - the highest reported in-device electro-optic coefficient of $325 \mathrm{pm} / \mathrm{V}$. The measurement agrees well with the two state model (transition wavelength of $1075 \mathrm{~nm}$, line width of $50 \mathrm{~nm}$ ) shown by the red dashed line in Fig. 3. While plots (a) and (b) indicate the strength of the light matter interaction, the modulator efficiency is ultimately defined by the ratio between loss and phase shift. $\alpha U_{\pi} L$ is an experimental figure of merit describing this ratio. It indicates the associated propagation losses of a phase modulator with a $\pi$-voltage of $1 \mathrm{~V}$. Fig. 2(c) shows this FOM over the wavelength range of interest. A decrease by almost a factor two can be observed and the decrease follows well the trend line (red dashed line) obtained from modal analysis and the two-state model. The losses were determined based on ellipsometry measurements of the materials' properties and on SEM images of the exact waveguide structure.

\section{Conclusion}

We showed that plasmonics allow to exploit the resonances of an OEO material resulting in an overall performance enhancement of electro-optic modulators. This approach results in a two times higher efficiency. The increased performance is also expected form other OEO-materials such as DLD-164 [8]. Thus, the reported voltage-length product of $40 \mathrm{~V} \mu \mathrm{m}$ for plasmonic Mach-Zehnder modulator can decrease to values well below $20 \mathrm{~V} \mu \mathrm{m}$ by employing the material resonances. Further, our experiments suggest that the engineering of novel OEO-materials is no longer bound to the requirement of low-loss organic molecules. Thus, designing can solely focus on maximizing the electrooptic coefficient which could bring us much closer to electro-optic coefficients as high as $1000 \mathrm{pm} / \mathrm{V}$.

We would like to acknowledge pratially funding by the ERC grant PLASILOR (640478), by the EU Project PLASMOFAB (688166), by the National Science Foundation (NSF) (DMR-1303080) and by the Air Force Office of Scientific Research (FA9550-15-1-0319).

\section{References}

[1]R. S. Weis et al., "Lithium niobate: Summary of physical properties and crystal structure," Applied Physics A, vol. 37, no. 4 pp. 191203,(1985).

[2]L. R. Dalton et al., Organic Electro-Optics and Photonics: Molecules, Polymers and Crystals: Cambridge University Press, 2015.

[3]D. A. B. Miller, "Attojoule Optoelectronics for Low-Energy Information Processing and Communications; a Tutorial Review," J. Lightwave Technol., vol. PP, no. 99 pp. 1-1,(2017).

[4]M. Lee et al., "Broadband modulation of light by using an electro-optic polymer," Science, vol. 298, no. 5597 pp. 1401-1403,(2002).

[5]C. Wang et al., "Nanophotonic lithium niobate electro-optic modulators," arXiv preprint arXiv:1701.06470, (2017).

[6]J. Leuthold et al., "Silicon-Organic Hybrid Electro-Optical Devices," Selected Topics in Quantum Electronics, IEEE Journal of, vol. 19, no. 6 pp. 114-126,(2013).

[7]S. A. Maier, Plasmonics: Fundamentals and Applications: Springer US, 2007.

[8]C. Haffner et al., "Plasmonic Organic Hybrid Modulators: Scaling Highest Speed Photonics to the Microscale," Proceedings of the IEEE, vol. 104, no. 12 pp. 2362-2379,(2016).

[9]W. Heni et al., "Nonlinearities of organic electro-optic materials in nanoscale slots and implications for the optimum modulator design," Opt. Express, vol. 25, no. 3 pp. 2627-2653,(2017).

[10]C. Haffner et al., "All-plasmonic Mach-Zehnder modulator enabling optical high-speed communication at the microscale," Nat Photon, vol. 9 , no. 8 pp. 525-528,(2015).

[11]S. Mossman et al., "Fundamental limits on the electro-optic device figure of merit," J. Opt. Soc. Am. B, vol. 33, no. 12 pp. E109-E120,(2016).

[12]J. Tian et al., "Broadband high-efficiency surface-plasmon-polariton coupler with silicon-metal interface," Applied Physics Letters, vol. 95, no. 1 p. 013504,(2009).

[13]D. L. Elder et al., "University of Washington", are prepering a manuscript to be called "Electro-optic chromophores with rigid aromatic bridge-protecting units"," (2017). 